\title{
Measurement of Downstream Charge Transport During Plasma Cleaning of Vacuum Chambers
}

\author{
C. A. Moore ${ }^{1}$ \\ ${ }^{1 .}$ XEI Scientific, Redwood City, CA, USA
}

Vacuum chambers suffer deleterious effects from the presence of adventitious hydrocarbons (AHCs). These AHCs originate from various sources including samples under analysis and can cause distortion of both images (blurring) as well compositional detection (via interference with secondary signals). A compounding factor is that AHCs typically possess very fast surface mobility such that a cleaned surface begins to show measureable re-contamination soon afterward (ones of hours). A common method to remediate AHC contamination in situ is through the use of plasma-based methods wherein an electrical discharge is ignited in a suitable precursor gas and the plasma products perform cleaning. Because of the sensitive nature of parts typically found in these tools it is highly desirable to utilize plasma products that are electrically neutral but chemically reactive. To achieve this goal it is usually arranged to have a plasma generation volume on an upstream port of the microscopy tool and to allow neutral plasma products flow downstream to work-pieces. Charged species more than $10 \mathrm{~cm}$ from the plasma are assumed not to occur, and we have measured negligible charge collection in hydrogen discharges using a Langmuir probe [1] at 200-600 $\mathrm{mT}$ under typical safe plasma cleaning powers (20 W maximum)

Recent trends [2] in commercial plasma decontamination equipment has been to lower plasma pressures as this increases the inelastic mean free path (MFP) [3] and decreases scattering to chamber walls, in turn leading to both order-of-magnitude increases in cleaning rates near $(<20 \mathrm{~cm})$ the plasma source but also measureable cleaning rates over $0.5 \mathrm{~m}$ away that at higher pressures were too low to reliably measure. Despite these considerable advantages, it does beg the question: if cleaning at lower pressures improves cleaning by chemically reactive neutrals, shouldn't all plasma products, including charged species, also reach farther into the chamber? And if so, at what magnitude and now does this depend on pressure?

To address this issue we fabricated a copper collection electrode and mounted it $20 \mathrm{~cm}$ from a plasma operating at $20 \mathrm{~W}$ (see Figure 1). The current collected by this thin plate was dissipated through a 100 $\mathrm{kOhm}$ resistor to ground and the resulting voltage was recorded by an oscilloscope. Small positive and negative biases on the copper plate had no effect on the signal, thus indicating there was no plasma discharge surrounding this piece. The measured signal in all cases consisted of small AC signal at the plasma drive frequency $(13.56 \mathrm{MHz})$ with a large positive DC offset. The AC signal was attributed to radiated noise while the DC signal was estimated to originate from charge transport. The positive nature of the DC signal indicates that the copper paddle was either collecting positive ions (likely) or emitting electrons (not likely).

We examined collected current as a function of chamber pressure and these results are shown in Figure 2. Above 100 mTorr where only a roughing pump was used we observe a pressure dependence that is very similar to that of a MFP curve. Below $100 \mathrm{mTorr}$ a turbo pump was also used, and because of loading by these relatively high pressures its rotational speed varied and results deviated from the results 
at higher pressure, indicative of a gas flow dependence of the charge transport. In all we conclude that charge transport occurs during plasma cleaning and its magnitude is inversely proportional to pressure, thus care must be exercised in environments where deposition of charge may be problematic.

\section{References:}

[1] Langmuir probe data in oxygen-containing plasmas are fraught with artifactual content and thus difficult to interpret, thus hydrogen was used as a proxy.

[2] C.A. Moore, Sematech Workshop on Nanodefects, Albany, NY, November 2012.

[3] That is, inelastic MFP is the mean travel distance until an inelastic collision occurs. Typical gases used in plasma cleaning are dominated by elastic gas phase collisions that serve mainly to scatter cleaning species in a diffusive manner.

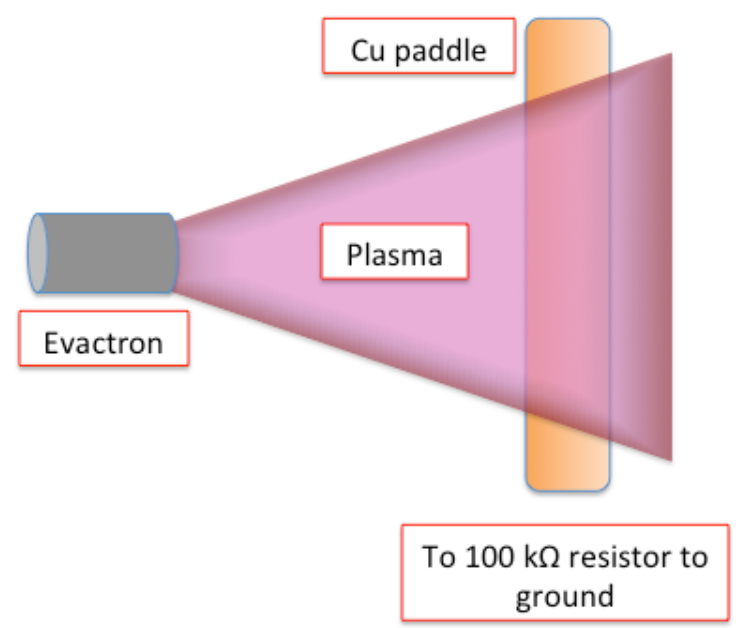

Figure 1. Experimental set-up for measurement of downstream charge transport from plasma system.

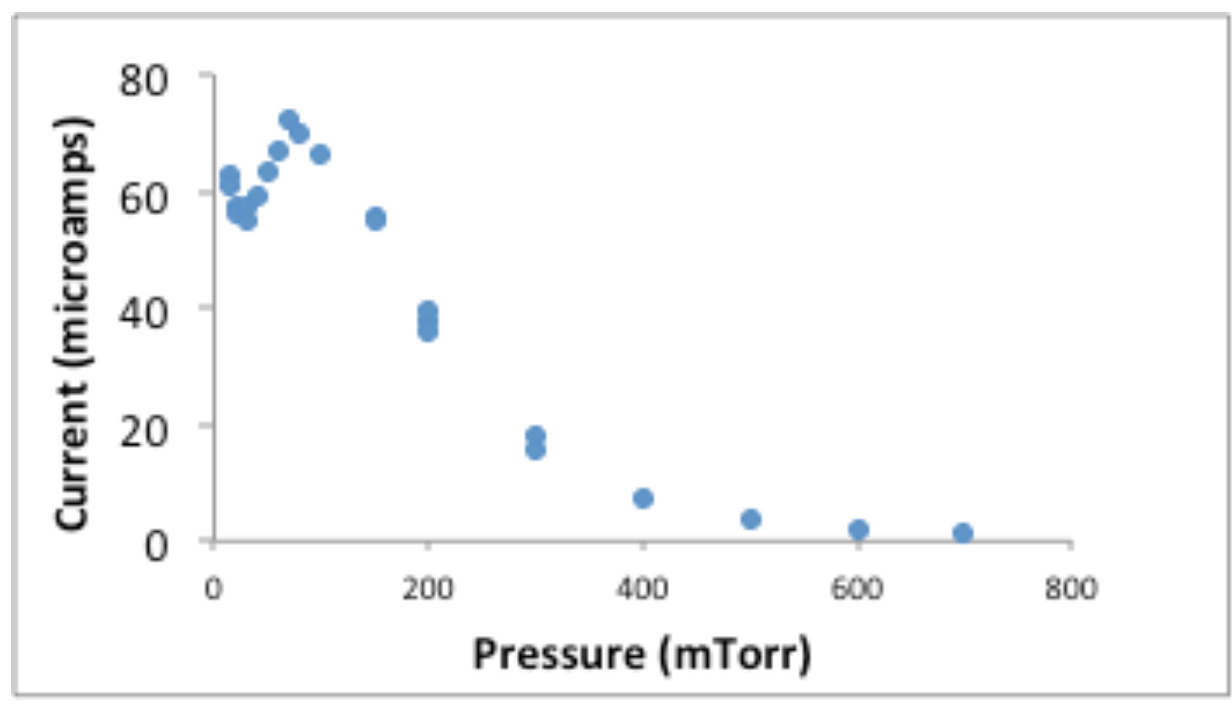

Figure 2. Collected current as a function of pressure. Below 100 mTorr the turbo pump was active but at uncontrolled rotation speeds. 